\title{
Anisotropic superconductivity and quantum oscillations in the layered dichalcogenide TaSnS 2
}

\author{
Manuel Feig, ${ }^{1,2}$ Michael Baenitz, ${ }^{2}$ Matej Bobnar, ${ }^{2}$ Klaus Lüders, ${ }^{2,3}$ Marcel Naumann, ${ }^{2}$ Walter Schnelle, ${ }^{2}$ Sergiy Medvediev, ${ }^{2}$ \\ K. M. Ranjith ${ }^{\circ},{ }^{2}$ Elena Hassinger, ${ }^{2}$ Tina Weigel, ${ }^{1}$ Dirk C. Meyer, ${ }^{1}$ Andreas Leithe-Jasper, ${ }^{2}$ Jens Kortus ${ }^{4},{ }^{4}$ \\ and Roman Gumeniuk (1, $1,2, *$ \\ ${ }^{1}$ Institut für Experimentelle Physik, TU Bergakademie Freiberg, Leipziger Straße 23, 09596 Freiberg, Germany \\ ${ }^{2}$ Max-Planck-Institut für Chemische Physik fester Stoffe, Nöthnitzer Straße 40, 01187 Dresden, Germany \\ ${ }^{3}$ Fachbereich Physik, Freie Universität Berlin, Arnimallee 14, 14195 Berlin, Germany \\ ${ }^{4}$ Institut für Theoretische Physik, TU Bergakademie Freiberg, Leipziger Straße 23, 09596 Freiberg, Germany
}

(Received 21 September 2020; revised 23 October 2020; accepted 6 November 2020; published 1 December 2020)

\begin{abstract}
$\mathrm{TaSnS}_{2}$ single crystal and polycrystalline samples are investigated in detail by magnetization, electrical resistivity, and specific heat as well as Raman spectroscopy and nuclear magnetic resonance (NMR). Studies are focused on the temperature and magnetic field dependence of the superconducting state. We determine the critical fields for both directions $B \| c$ and $B \perp c$. Additionally, we investigate the dependence of the resistivity, the critical temperature, and the structure through Raman spectroscopy under high pressure up to $10 \mathrm{GPa}$. At a pressure of $\approx 3 \mathrm{GPa}$ the superconductivity is suppressed below our minimum temperature. The Sn NMR powder spectrum shows a single line which is expected for the $\mathrm{TaSnS}_{2}$ phase and confirms the high sample quality. Pronounced de Haas-van Alphen oscillations in the ac susceptibility of polycrystalline sample reveal two pairs of frequencies indicating coexisting small and large Fermi surfaces. The effective mass of the smaller Fermi surface is $\approx 0.5 m_{\mathrm{e}}$. We compare these results with the band structures from DFT calculations. Our findings on $\mathrm{TaSnS}_{2}$ are discussed in terms of a quasi-two-dimensional BCS superconductivity.
\end{abstract}

DOI: 10.1103/PhysRevB.102.214501

\section{INTRODUCTION}

Superconductors with layered crystal structures have attracted the interest of researchers since the earlier 1960's. This is mainly due to their anisotropic superconducting properties and the associated very high critical fields [1-4]. In the 1980 s, this research got a strong impetus with the discovery of high- $T_{\mathrm{c}}$ superconductivity (SC) in cuprates [4-9]. In the last decade, the investigation of such quasi-two-dimensional (2D) materials invigorated after reports about anisotropic superconductivity in $\mathrm{BiS}_{2}$-based compounds and Fe pnictides [10-16].

The layered crystal structure is also characteristic for transition-metal dichalcogenides (TMD). Therefore, their properties are frequently said to be reminiscent of those of high- $T_{\mathrm{c}}$ cuprates and iron-based pnictides [4,17]. The most common polymorphic modifications of TMDs are the trigonal $1 T$ - and hexagonal $2 \mathrm{H}$-polytypes. Interestingly, pristine TMDs with the $1 T$-polytype structure were found to be mostly semiconducting or semimetallic, while SC is never observed for them at ambient pressure. The reason is a charge density wave $(\mathrm{CDW})$ instability of these materials which is eventually

\footnotetext{
*roman.gumeniuk@physik.tu-freiberg.de
}

Published by the American Physical Society under the terms of the Creative Commons Attribution 4.0 International license. Further distribution of this work must maintain attribution to the author(s) and the published article's title, journal citation, and DOI. Open access publication funded by the Max Planck Society. suppressed by application of high pressure. In contrast, some $2 H$-TMDs do not show this instability and frequently become superconducting at ambient pressure and low temperatures, as, e.g., $2 H-\mathrm{NbS}_{2}\left(T_{\mathrm{c}}=5.8 \mathrm{~K}\right)[18], 2 H-\mathrm{TaS}_{2}\left(T_{\mathrm{c}}=0.8 \mathrm{~K}\right)$ $[19,20], 2 H-\mathrm{NbSe}_{2}\left(T_{\mathrm{c}}=7.0 \mathrm{~K}\right)[21]$.

Interestingly, there are several routes to enhance $T_{\mathrm{c}}$ of TMDs: (i) by increasing the transition-metal content, as it is observed for $\mathrm{TaSe}_{2}\left(T_{\mathrm{c}}=0.14 \mathrm{~K}\right)[22,23]$ and $\mathrm{Ta}_{1.06} \mathrm{Se}_{2}\left(T_{\mathrm{c}}=\right.$ $3.2 \mathrm{~K})$ [24]; (ii) by third elements, e.g., by substitutions on the transition-metal site of pristine $2 \mathrm{H}$-TMD. One example, $\mathrm{TaSe}_{2}$ doped with $\mathrm{Nb}$ (i.e., $\mathrm{Ta}_{0.8} \mathrm{Nb}_{0.2} \mathrm{Se}_{2}$ ) reveals a $T_{\mathrm{c}}$ up to $2.01 \mathrm{~K}$ $[25,26]$; (iii) intercalation of some organic molecules into TMD crystals by soft chemistry methods. These species are located between the chalcogenide layers which are separated by a van der Waals gap [27]. The most prominent example is $\mathrm{TaS}_{2}$ (pyridine) $)_{1 / 2}$, a very anisotropic superconductor with strongly enhanced $T_{\mathrm{c}}=3.5 \mathrm{~K}[3,4,28]$.

(iv) Finally, there are various 'intercalation' compounds, where in the space between the layers metals as $\mathrm{Hg}$, In $\mathrm{Pb}, \mathrm{Sn}$, etc. but also transition metals are incorporated [17]. Several such $\operatorname{Ta}_{x} S_{2}(x=1 / 4-1)$ materials are based on $2 H-\mathrm{TaS}_{2}$. Concerning superconductivity $\mathrm{TaS}_{2}$ intercalated with Pd reveals a $T_{\mathrm{c}}$ up to $4.2 \mathrm{~K}$ [29], whereas $\mathrm{Cu}$ [30] and $\mathrm{Ni}$ intercalation [31] lead to maximum $T_{\mathrm{c}}=4.5 \mathrm{~K}$ and $T_{\mathrm{c}}=4.15 \mathrm{~K}$, respectively.

Among the numerous intercalated layered TMDs with the basic $2 H-\mathrm{TaS}_{2}$ structure [32,33], $\mathrm{TaSnS}_{2}$ (SG: $P 6_{3} / m m c, a \approx$ $3.3 \AA, c \approx 17.4 \AA$ ) attracted special attention due to its rare structural arrangement, which is characterized by the linear coordination of the intercalated Sn atoms (i.e., appearance of 
linear S-Sn-S units), which connects the hexagonal $2 \mathrm{H}-\mathrm{TaS}_{2}$ layers [34,35]. Magnetic susceptibility and transport measurements revealed $\mathrm{TaSnS}_{2}$ to be a diamagnetic metal with a superconducting transition temperature $T_{\mathrm{c}}=2.95 \mathrm{~K}$ and the normal state transport properties to be mainly determined by the $\mathrm{Sn} 5 p_{x} p_{y}$ band [36-38]. The average valency $\mathrm{Sn}^{1+}$, which arises from rapid valence fluctuations between $\mathrm{Sn}^{0}$ and $\mathrm{Sn}^{2+}$ [38,39], was deduced from photoelectron- [37-39], Mössbauer- [40-43], and NMR-spectroscopy [44] measurements. This unusual valence state was confirmed by band structure calculations $[38,45,46]$ and is associated with electron donation of the intercalated Sn until the Ta $d_{z^{2}}$ band is completely filled [38]. Powder neutron and x-ray diffraction [34] as well as single crystal x-ray diffraction [35] confirmed the presence of only one crystallographic $\mathrm{Sn}$ site in $\mathrm{TaSnS}_{2}$. These studies revealed a rather long $\mathrm{Sn}-\mathrm{S}$ distance of about $2.8 \AA$, which again could be explained by the low charge of Sn.

Superconductivity in $\mathrm{TaSnS}_{2}$ was reported recently [47]. As expected, $\mathrm{TaSnS}_{2}$ is an anisotropic superconductor, where the second critical magnetic field $B_{c 2}^{\perp} \approx 300 \mathrm{mT}$ is much larger than that measured parallel to the $c$ direction $\left(B_{\mathrm{c} 2}^{\|} \approx\right.$ $20 \mathrm{mT}$ ). The same study reports $\mathrm{TaSnS}_{2}$ to be a SC with moderately strong electron-phonon coupling as well as a nodal line semimetal.

Recently, some TMDs gained attention as a possible realization of topological SC. Candidate materials are Weyl type II systems like $\mathrm{MoTe}_{2}$ [48] and $\mathrm{WSe}_{2}$ (SC is induced by applying pressure) [49]. The ternary compounds $\mathrm{TaSnS}_{2}$ [47], $\mathrm{TaPbS}_{2}$ [50], and $\mathrm{TaPbSe}_{2}$ [51-54] are discussed in the same context as superconducting topological nodal line semimetals. The signatures of such a behavior are band structures (spin-orbit coupling is not considered) exhibiting several band crossings near the Fermi level $\left(E_{\mathrm{F}}\right)$, which form three nodal lines in the $k_{z}=0$ plane, resulting in drumheadlike surface states. However, the crystal structure of $\mathrm{TaPbSe}_{2}$ is noncentrosymmetric. With this structural difference, the question remains open if superconductivity observed in both systems has to be associated to the presence of topological line node states at the Fermi level or to residual trivial states forming a flat band near the $E_{\mathrm{F}}$. Contrasting that, recent findings ( $\mathrm{SC}$ at $T_{\mathrm{c}}=2.6 \mathrm{~K}$ ) on the related centrosymmetric nodal line semimetal $\mathrm{TaPbS}_{2}$ [50] are reminiscent of our $\mathrm{TaSnS}_{2}$ results.

These new and controversial findings triggered us to perform a comprehensive study on $\mathrm{TaSnS}_{2}$ single crystals and polycrystalline material. Anisotropic SC is studied as a function of temperature and magnetic field via magnetization, specific heat, and electrical transport, and results obtained are in good agreement with findings from Ref. [47]. $\mathrm{TaSnS}_{2}$ is identified as an anisotropic BCS-like superconductor. Investigations beyond bulk properties presented here are resistivityand Raman spectroscopy measurements under pressure, Snnuclear magnetic resonance (NMR) as well as the evaluation of quantum oscillations in the dc field dependent ac magnetic susceptibility. Pronounced de Haas-van Alphen oscillations confirm the presence of light charge carriers in the semimetal $\mathrm{TaSnS}_{2}$. Resistivity measurements and Raman studies under high pressure reveal the suppression of superconductivity with pressure and suggest a pressure-induced structural phase transition.

\section{EXPERIMENTAL}

To synthesize polycrystalline $\mathrm{TaSnS}_{2}$ the elemental powders (Sn: Chempur, 99.995 wt.\%, Ta: Chempur, 99.9 wt.\%, $\mathrm{S}$ : Chempur, 99.5 wt.\%) were mixed with iodine $(40 \mathrm{mg}$ per $\mathrm{g} \mathrm{TaSnS}{ }_{2}$ ) under protective Ar-atmosphere (MBraun glove box system $\left.\left[p\left(\mathrm{H}_{2} \mathrm{O}\right)<1 \mathrm{ppm} ; p\left(\mathrm{O}_{2}\right)<1 \mathrm{ppm}\right]\right)$, pressed into a pellet and enclosed in an evacuated quartz ampoule. The heat treatment was performed in two steps: first heating up to $450{ }^{\circ} \mathrm{C}$ within $24 \mathrm{~h}$ (kept at this temperature for another $24 \mathrm{~h}$ ) and second heating up to $800^{\circ} \mathrm{C}$ within $12 \mathrm{~h}$ and annealing for 7 days. The obtained powder was compacted applying spark plasma sintering (SPS-15 ET setup, Dr. Sinter Lab, Japan). It was also used for single crystal growth by chemical vapor transport (CVT). For this purpose, $\mathrm{TaSnS}_{2}$ was mixed with chlorine ( $4 \mathrm{mg} \mathrm{cm}^{-3}$ of $\mathrm{NH}_{4} \mathrm{Cl}$ [AlfaAesar, 99.999 wt.\%] calculated for total ampoule volume of $\approx 8 \mathrm{~cm}^{3}$ ) as transport agent and placed in an evacuated quartz ampoule $(10 \mathrm{~cm} \times$ $1.6 \mathrm{~cm}$ ). The temperature gradient from $925^{\circ} \mathrm{C}$ (source) to $800{ }^{\circ} \mathrm{C}$ (sink) in the ampoule was kept for 12 days. Further it was cooled $\left(60 \mathrm{~K} \mathrm{~h}^{-1}\right)$ down to $300{ }^{\circ} \mathrm{C}$ and finally air cooled to room temperature. The obtained crystals were thin plates with dimensions of $\approx 1 \times 1 \times 0.1 \mathrm{~cm}^{3}$.

The samples (i.e., polycrystalline powder, compacted polycrystalline SPS sample, and single crystals) were characterized by powder x-ray diffraction (XRD) on a Guinier camera $\mathrm{G} 670, \mathrm{Cu} K \alpha_{1}$ radiation. High-resolution powder $\mathrm{XRD}$ data were collected at room temperature at BM20 beamline of the European Synchrotron Radiation Facility (ESRF) $\left(\lambda=0.45923 \AA, 2 \theta_{\max }=32^{\circ}\right)$ on powder enclosed in a quartz capillary with an outer diameter of $0.3 \mathrm{~mm}$. Phase analysis was performed with the WinXpow [55] software package. Single crystal x-ray diffraction was performed on a Bruker AXS D8Quest single crystal diffractometer [56] with Mo $K \alpha$-radiation $\lambda=0.71076 \AA$, a Photon 100 detector and a TRIUMPH monochromator. Integration and correction was performed with the APEX software [57]. The lattice parameters by least-squares fitting and crystal structure were refined with WinCSD [58].

Magnetization measurements are performed on single crystals using a standard magnetometer (MPMS XL7, Quantum Design) and ac susceptibility measurements are conducted on the powder sample (ACMS option, PPMS14, Quantum Design). The electrical resistivity was measured on a SPS sample down to $1.9 \mathrm{~K}$ and the specific heat on a SPS sample down to $0.35 \mathrm{~K}$ as well as on a single crystal in two orientations (with the applied magnetic field parallel and perpendicular to the $c$ axis) in a commercial system equipped with a ${ }^{3} \mathrm{He}$ cooling stage (ACT and HC options, PPMS, Quantum Design).

Field swept ${ }^{117,119}$ Sn NMR measurements on polycrystalline material were performed using a Tecmag spectrometer employing standard pulse techniques at a given temperature of $5 \mathrm{~K}$ and at $35 \mathrm{MHz}$. The spectral intensity was obtained by integrating the spin echo in the time domain. In order to determine the Larmor field (NMR shift zero) we measured $\mathrm{SnO}_{2}$ powder as a nonmagnetic reference with ${ }^{119} \mathrm{~K}=0.5 \%$ [59].

For high-pressure Raman spectroscopy and electrical resistivity measurements, a screw-clamped diamond anvil cell with 
a $500 \mu \mathrm{m}$ culet was used. The tungsten gasket was insulated with a cubic BN/epoxy mixture. A single crystal sample of suitable size $(\sim 120 \mu \mathrm{m} \times 120 \mu \mathrm{m} \times 30 \mu \mathrm{m})$ was cut and placed into the central hole of the gasket filled with $\mathrm{NaCl}$ as a pressure-transmitting medium along with a ruby chip for pressure calibration. The electrical leads were fabricated from $5 \mu \mathrm{m}$ thick Pt foil and attached to the sample in a van der Pauw configuration. Electrical resistivity was measured in the temperature range $1.8-300 \mathrm{~K}$ using a direct current source and nanovoltmeter. The inaccuracy in the $T_{\mathrm{c}}$ in high-pressure experiments is estimated from calibration of our high-pressure measurement setup by measurements of pressure dependence of $T_{\mathrm{c}}$ of lead.

Raman spectra were collected at room temperature in backscattering geometry using a customary confocal microRaman spectrometer equipped with $20 \times$ long-workingdistance objective, $\mathrm{HeNe}$ laser as the excitation source, and a single-grating spectrograph with $1 \mathrm{~cm}^{-1}$ resolution.

Full potential linear augmented plane wave (LAPW) calculations were performed using the WIEN2k package [60]. We used the generalized gradient approximation [61] in the form of PBEsol [62] for the exchange-correlation potential. It is furthermore essential to use a very fine mesh in $k$ space; we employed a $39 \times 39 \times 21 \mathrm{mesh}$, corresponding to 1617 nonequivalent $k$ points. To get the areas of the orbits (given here in Tesla units) with sufficient accuracy we used an integration engine built in the SURFER program [63], which internally interpolates the integrand with splines. The results reported here include the effect of spin-orbit coupling.

\section{RESULTS AND DISCUSSION}

\section{A. Crystal structure}

To check the quality of the synthesized $\mathrm{TaSnS}_{2}$ crystals single crystal XRD was performed. All peaks in the collected dataset were successfully indexed in hexagonal symmetry with unit cell parameters presented in Table I. The analysis of the extinction conditions indicated three possible space groups (SG): $P 6_{3} m c, P \overline{6} 2 c$, and $P 6_{3} / m m c$. The highest symmetrical SG $P 6_{3} / \mathrm{mmc}$ was chosen for the first attempt to find a structural model. The atomic coordinates obtained from direct methods (Table I) were consistent with those reported for $\mathrm{TaSnS}_{2}$ in Ref. [35]. The crystallographic details of the further performed refinement, atomic coordinates, equivalent and anisotropic displacement parameters are collected in Table I.

The obtained crystal structure is considered in the literature $[34,35]$ as an intercalation of $\mathrm{Sn}$ layers in $2 \mathrm{H}-\mathrm{TaS}_{2}$ (Fig. 1). It is also characterized by interatomic distances close to the sums of atomic radii $\left(r_{\mathrm{Ta}}=1.43 \AA, r_{\mathrm{Sn}}=1.41 \AA, r_{\mathrm{S}}=\right.$ $1.04 \AA$ ). The elongated (by $\approx 14 \%$ ) $\mathrm{Sn}-\mathrm{S}$ contacts allow enlarged thermal displacements $B_{\text {eq }}$ which are much larger in comparison with the displacements of other atoms (Table I).

To confirm the obtained structural model NMR powder spectra were measured. They revealed two lines (Fig. 2), which could be assigned to the two predominant NMR active (spin 1/2) Sn isotopes. The NMR shift of those lines is positive and small (about $+0.4 \%$, in reasonable agreement with Ref. [44]) which indicates a small residual density of states at the Fermi level as expected for a semimetal. The
TABLE I. Crystallographic data and shortest interatomic distances for $\mathrm{TaSnS}_{2}\left\{\mathrm{SG} \mathrm{P6} 6_{3} / m m c, Z=2\right.$, Ta in $2 c(1 / 3,2 / 3,1 / 4)$, $\mathrm{Sn}$ in $2 a(0,0,0), \mathrm{S}$ in $4 e[0,0,0.16036(8)]$.

Lattice parameters ${ }^{1}$

\begin{tabular}{|c|c|}
\hline$a(\AA)$ & $3.30648(1)$ \\
\hline$c(\AA)$ & $17.4416(1)$ \\
\hline$V\left(\AA^{3}\right)$ & $165.14(1)$ \\
\hline$\rho\left(\mathrm{g} \mathrm{cm}^{-3}\right)$ & 7.31(1) \\
\hline $\operatorname{Mo} K_{\alpha}, \lambda(\AA)$ & 0.71076 \\
\hline $2 \theta_{\max }\left({ }^{\circ}\right)$ & 81.74 \\
\hline Minimum $h, k, l$ & $-6,-5,-31$ \\
\hline Maximum $h, k, l$ & $6,6,31$ \\
\hline$N(h k l)_{\text {measured }}$ & 6140 \\
\hline$N(h k l)_{\text {unique }}$ & 254 \\
\hline$N(h k l)_{\text {observed }}\left(F_{h k l} \geqslant 2 \sigma(F)\right)$ & 5334 \\
\hline$R_{\text {int }} / R_{\sigma}$ & $0.082 / 0.069$ \\
\hline Refined parameters & 12 \\
\hline Extinction coefficient & $0.0101(6)$ \\
\hline Goodness of fit, $S$ & 1.010 \\
\hline$R_{F} / w R_{F^{2}}^{2}$ & $0.039 / 0.040$ \\
\hline \multicolumn{2}{|l|}{ Min. and max. residual } \\
\hline electronic density $\left(e \AA^{-3}\right)$ & $-4.5 ;+6.1$ \\
\hline$B_{\mathrm{eq}}{ }^{3}, B_{11}, B_{33}$ for $\mathrm{Ta}^{4}$ & $0.29(1), 0.28(1), 0.32(1)$ \\
\hline$B_{\mathrm{eq}}{ }^{3}, B_{11}, B_{33}$ for $\mathrm{Sn}^{4}$ & $1.45(1), 1.69(2), 0.96(2)$ \\
\hline$B_{\mathrm{eq}}{ }^{3}, B_{11}, B_{33}$ for $\mathrm{S}^{4}$ & $0.45(2), 0.48(3), 0.40(4)$ \\
\hline$d(\mathrm{Ta}-6 \mathrm{~S}) ; d(\mathrm{Ta}-6 \mathrm{Ta}),(\AA)$ & $2.4675(9), 3.3065(1)$ \\
\hline$d(\mathrm{Sn}-2 \mathrm{~S}) ; d(\mathrm{~S}-1 \mathrm{~S}),(\AA)$ & $2.797(1), 3.127(2)$ \\
\hline \multicolumn{2}{|l|}{${ }^{1}$ Powder XRD data } \\
\hline \multicolumn{2}{|c|}{$\begin{array}{l}{ }^{2} R=\left[\Sigma\left(\left|F_{\mathrm{o}}\right|-\left|F_{\mathrm{c}}\right|\right)\right] / \Sigma\left|F_{\mathrm{o}}\right| ; \quad w=0.1176 \lg \left(F_{\mathrm{o}}\right)^{4} ; \quad w R=\left\{\Sigma\left[w\left(F_{\mathrm{o}}^{2}-\right.\right.\right. \\
\left.\left.\left.F_{\mathrm{c}}^{2}\right)^{2}\right] / \Sigma\left[w\left(F_{\mathrm{o}}^{2}\right)^{2}\right]\right\}^{1 / 2} \\
{ }^{3} B_{\mathrm{eq}}=1 / 3\left[B_{11} a^{* 2} a^{2}+\ldots 2 B_{23} b^{*} c^{*} b c \cos \alpha\right] \\
{ }^{4} B_{22}=B_{11}, B_{12}=1 / 2 B_{11}, B_{13}=B_{23}=0\end{array}$} \\
\hline
\end{tabular}

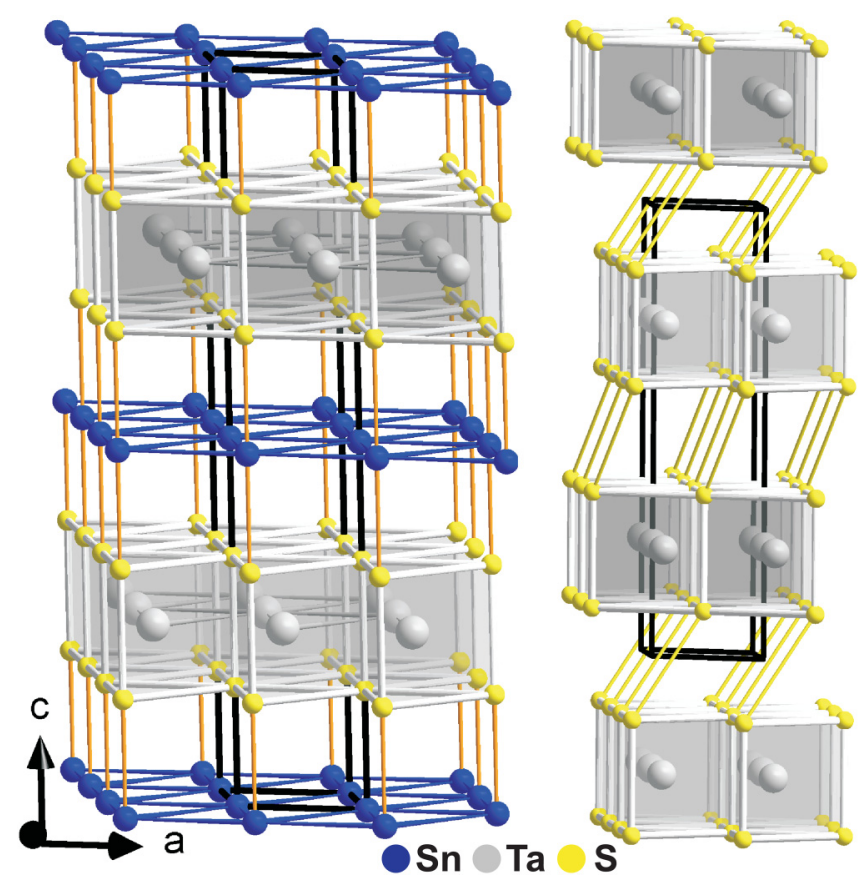

FIG. 1. The structural relationship between $\mathrm{TaSnS}_{2}$ (left) and $\mathrm{TaS}_{2}$ (right) illustrates the intercalation of Sn layers in $2 H-\mathrm{TaS}_{2}$. 


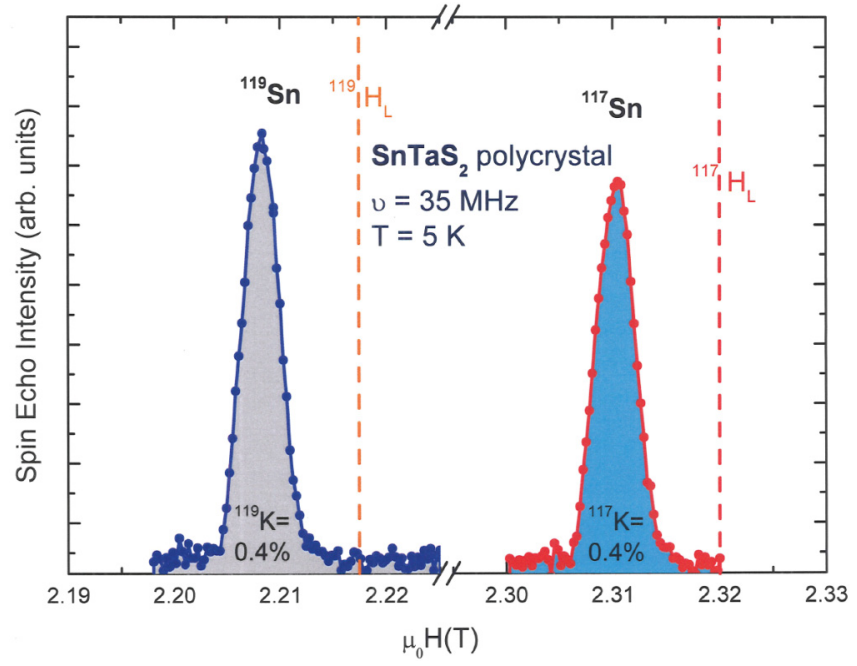

FIG. 2. Sn NMR field sweep spectra of the $\mathrm{TaSnS}_{2}$ polycrystalline sample. The vertical dashed lines indicate the Larmor fields for the two Sn isotopes.

difference in the shift value to Ref. [44] (determined at about $0.5 \mathrm{~T}$ ) might originate from $\mathrm{dHvA}$ oscillations in the shift itself. This is frequently found in semimetals [64]. There are no further NMR lines found which evidences that there is no additional line associated to other phases like $\mathrm{Sn}_{1 / 3} \mathrm{TaS}_{2}$ (with ${ }^{119} \mathrm{~K} \simeq 0.08 \%$ ) [44]. The line positions (resonance fields) and the absolute intensities match with the prediction from the gyromagnetic ratios and the natural abundance of the respective Sn nuclei. So from our NMR study we have clear evidence for a well defined stoichiometry in the powder material and we could rule out other Sn containing foreign phases (like $\mathrm{Sn}_{1 / 3} \mathrm{TaS}_{2}$ ). This observation is in good agreement with the results of phase analysis performed on high-resolution powder XRD patterns (see Fig. S1 in Supplemental Material [65]).

\section{B. Magnetic measurements}

The temperature dependence of the critical fields $B_{\mathrm{c} 1}(T)$ and $B_{\mathrm{c} 2}(T)$ was determined from temperature- and fielddependent (ZFC-FC) isothermal magnetization measurements as well as ac susceptibility (for more details see Supplemental Material [65]). Figure 3 shows the transition curves at a nominal magnetic field $B=0.5(2) \mathrm{mT}$ for the orientation $B \perp c$ [Fig. 3(a)] and $B \| c$ [Fig. 3(b)], respectively. In the case of $B \perp c$, the resulting susceptibility leads to a maximum superconducting volume fraction of $\approx 82 \%$. For $B \| c$ the measured magnetization is considerably enhanced due to the large demagnetization factor of the platelike sample. We define critical temperatures $T_{\mathrm{c}}$ by the onset of the diamagnetic transition as indicated by arrows in Fig. 3. For the lowest applied field the $T_{\mathrm{c}}$ is $3.0(1) \mathrm{K}$. Figure 4 shows further ZFCFC transition curves for both field orientations. The resulting $B_{\mathrm{c} 2}(T)$ curves are plotted in Fig. 6 . The transition temperature extrapolated to zero field is $T_{\mathrm{c}}=3.04 \mathrm{~K}$.

One branch of an isothermal magnetization curve at $T=$ $2 \mathrm{~K}$ for the orientation $B \perp c$ is plotted in Fig. 5. It shows a nearly reversible behavior indicating weak flux pinning in the sample. A very similar behavior was found for $\mathrm{TaPbS}_{2}$
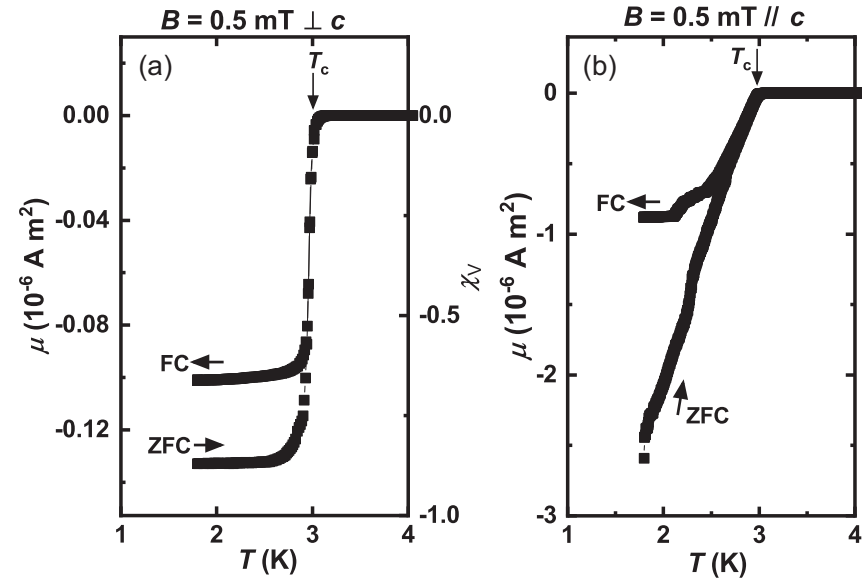

FIG. 3. Zero-field cooled (ZFC, shielding effect) and field cooled (FC, Meissner effect) susceptibility for $\mathrm{TaSnS}_{2}$ single crystals with a nominal field of $0.5 \mathrm{mT}$ applied in two directions: (a) $B \perp c$, (b) $B \|$ $c$.

single crystals [50]. In the inset the respective ZFC and FC values are also plotted showing excellent agreement. The visible deviation from linearity with increasing field allows the estimation of the lower critical field at this temperature: $B_{\mathrm{c} 1}^{\perp}(2 \mathrm{~K}) \approx 1.95 \mathrm{mT}$.

The temperature evolution of the direction dependent upper critical fields $B_{\mathrm{c} 2}^{\perp}$ and $B_{\mathrm{c} 2}^{\|}$estimated from the measurements of isothermal magnetization, electrical resistivity, and specific heat are depicted in Fig. 6. Interestingly, the results obtained from $c_{p}(T)$ and $\rho(T)$ measured on polycrystalline samples nicely coincide with those observed for $B \| c$ and $B \perp c$, respectively. Fitting $B \| c$ data to the Ginzburg-Landau (GL) relation: $B_{\mathrm{c} 2}(T)=B_{\mathrm{c} 2}(0)\left(1-t^{2}\right) /\left(1+t^{2}\right)$ with $t=$ $T / T_{\mathrm{c}}$ [66] the upper critical field is estimated as $B_{\mathrm{c} 2}^{\|}(0)=$ 27(2) $\mathrm{mT}$ (inset to Fig. 6).

The upward curvature of the $B_{\mathrm{c} 2}\left(T_{\mathrm{c}}\right)$ dependence is known for both doped [67] and intercalated [68] tantalum dichalcogenides, including $\mathrm{TaSnS}_{2}$ [47]. In the case of $\mathrm{TaPbSe}_{2}[68]$ it
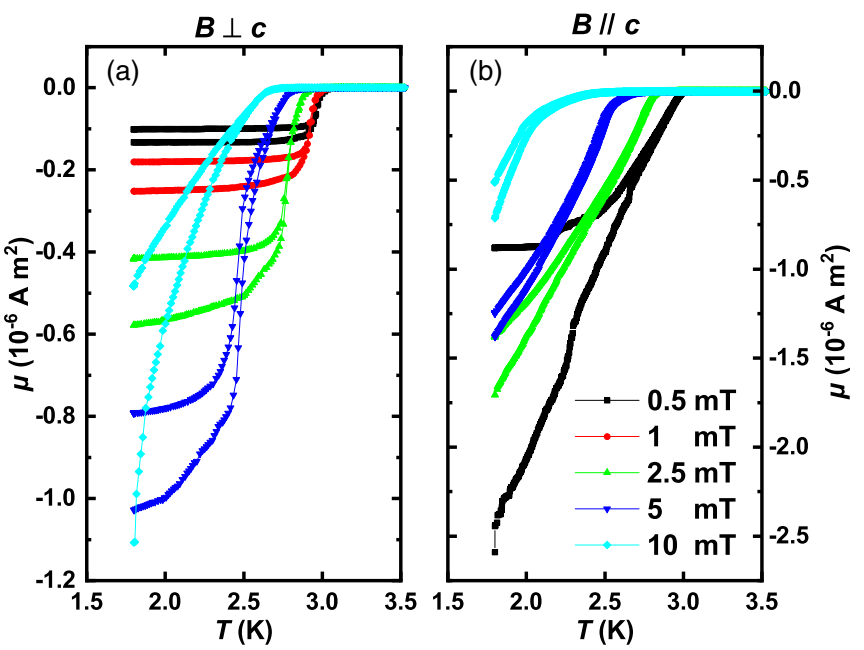

FIG. 4. Magnetic susceptibility of $\mathrm{TaSnS}_{2}$ single crystals with higher applied magnetic fields: (a) $B \perp c$ and (b) $B \| c$. 


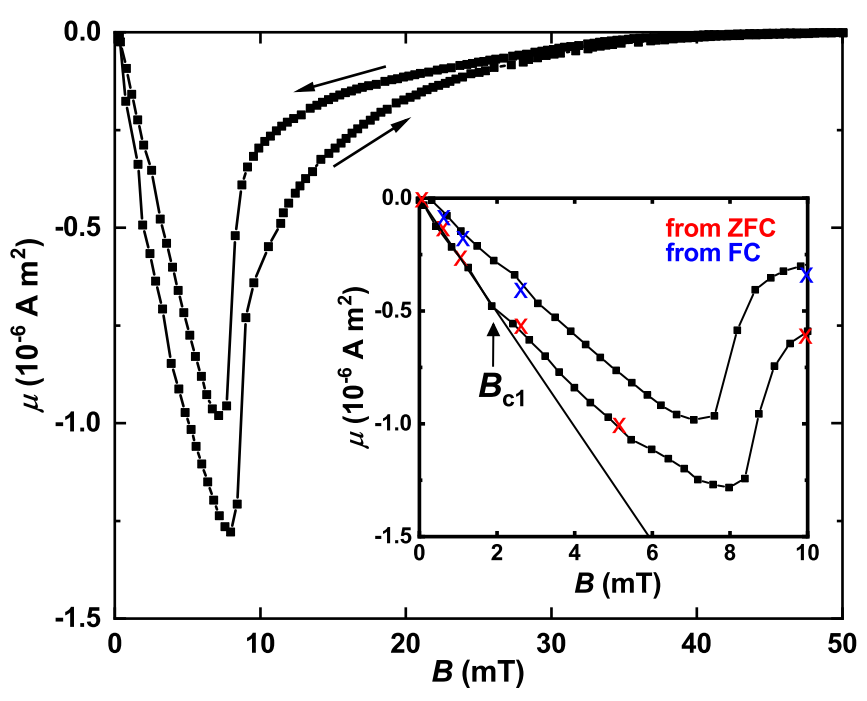

FIG. 5. Positive field branch of the magnetization curve of $\mathrm{TaSnS}_{2}$ for the orientation $B \perp c$ and the temperature $T=2 \mathrm{~K}$. The inset contains additionally the respective ZFC and FC values for comparison and the deviation from the first linear part to determine the lower critical field $B_{\mathrm{c} 1}$.

could be fitted with $B_{\mathrm{c} 2}(T)=B_{\mathrm{c} 2}(0)\left(1-t^{3 / 2}\right)^{3 / 2}$. However, this equation is reported to fail in the description of $B_{\mathrm{c} 2}^{\perp}\left(T_{\mathrm{c}}\right)$ for $\mathrm{TaSnS}_{2}$ [47]. As is seen from Fig. 6, this finding is also confirmed in our study. Interestingly, $B_{\mathrm{c} 2}(T)=B_{\mathrm{c} 2}(0)(1-$ $\left.t^{5 / 2}\right)^{5 / 2}$ with $B_{\mathrm{c} 2}^{\perp}(0)=170(10) \mathrm{mT}$ describes well the data deduced from the field dependent $\rho(T)$ measurements on the polycrystalline sample. Obviously, this critical field can be under/overestimated, taking into account the lack of the points for $T_{\mathrm{c}}<1.7 \mathrm{~K}$.

The anisotropy parameter of $\approx 6.3$ is calculated from the relation of two upper critical fields. The GL coherence lengths

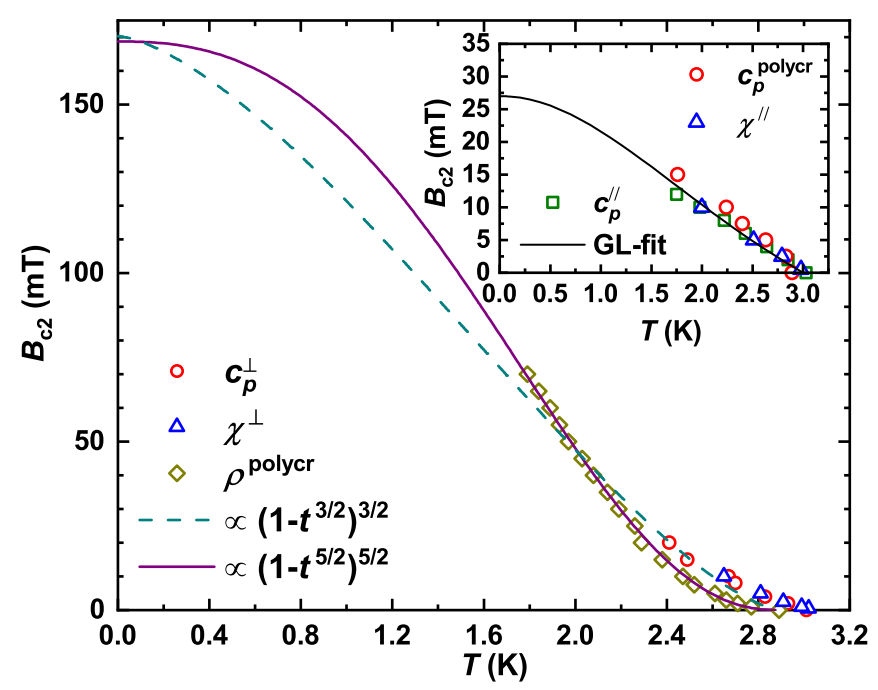

FIG. 6. Upper critical field $B_{\mathrm{c} 2}$ of $\mathrm{TaSnS}_{2}$ versus temperature from $c_{p}, \chi$, and $\rho$ measurements on polycrystalline (polycr) material as well as $c_{p}$ and $\chi$ measurements on single crystals. Inset: Detailed view on the data in the temperature range $1.6-3.0 \mathrm{~K}$. For the fits (lines) see the text.
$\xi_{\mathrm{GL}}^{\|}=17(2) \mathrm{nm}$ and $\xi_{\mathrm{GL}}^{\perp}=110(6) \mathrm{nm}$ were estimated using Eqs. (1) and (2), where $\Phi_{0}$ is the magnetic flux quantum, respectively.

$$
\begin{aligned}
& \xi_{\mathrm{GL}}^{\perp}=\sqrt{\frac{\Phi_{0}}{2 \pi B_{\mathrm{c} 2}^{\|}}} \\
& \xi_{\mathrm{GL}}^{\|}=\frac{\Phi_{0}}{2 \pi B_{\mathrm{c} 2}^{\perp} \xi_{\mathrm{GL}}^{\perp}}
\end{aligned}
$$

Using $B_{\mathrm{c} 1}^{\perp}(2 \mathrm{~K})$ from the magnetization measurements, the London penetration depth $\lambda^{\perp}=280(10) \mathrm{nm}$ has been determined by a numerical solution of the equation $B_{\mathrm{c} 1}^{\perp}=$ $\left(\Phi_{0} / 4 \pi\left(\lambda^{\perp}\right)^{2}\right) \ln \left(\lambda^{\perp} / \xi_{\mathrm{GL}}^{\perp}\right)$. Thus, the GL parameter $\kappa_{\mathrm{GL}}^{\perp}=$ $\lambda^{\perp} / \xi_{\mathrm{GL}}^{\perp}=2.5(2)$ indicates $\mathrm{TaSnS}_{2}$ to be a type-II superconductor.

Due to the positive curvature of the $B_{\mathrm{c} 2}^{\perp}\left(T_{\mathrm{c}}\right)$ curve, the anisotropy decreases to a value of $\approx 3$. Similarly low values have been reported for other doped (e.g., with $\mathrm{Nb}$ [69]) or intercalated with $\mathrm{Sn}$ [70], $\mathrm{Na}$ [71], Pd [72] as well as with organic molecules $[67,73]$ layer structured tantalum dichalcogenides. These observations are in agreement with the prediction by the dimensionality crossover model of Klemm et al. [74] developed for superconductor/insulator superlattices. It was also found in metal intercalated dichalcogenides $[72,26]$ and was explained by special Fermi surface properties. Also for other layered superconductors like graphite intercalation compounds (GICs) [75] and especially for the high- $T_{\mathrm{c}}$-cuprates $[6,7,16]$ positive curvatures were reported. It was speculated that such a behavior is a common feature for two-dimensional superconducting systems. However, there also exist distinct deviations from this rule, especially for GICs, where extended linearity of $B_{\mathrm{c} 2}(T)$ was reported [76]. The positive curvatures near $T_{\mathrm{c}}$ occasionally found in isotropic systems like superconducting fullerenes $[77,78]$ might originate from the presence of different phases with variations in the local $T_{\mathrm{c}}$. In view of such a variety of results further investigations are desired to find an appropriate model for the positive curvature analysis for $\mathrm{TaSnS}_{2}$.

\section{Electrical resistivity and specific heat}

The electrical resistivity $\rho(T)$ in different magnetic fields is measured on the SPS-compacted $\mathrm{TaSnS}_{2}$ sample (Fig. 7). In zero-field $\rho(T)$ decreases with decreasing temperature down to $\approx 3 \mathrm{~K}$ indicating $\mathrm{TaSnS}_{2}$ to be a metallic system. The residual resistivity ratio $\mathrm{RRR}=\rho^{300 \mathrm{~K}} / \rho_{0}=10.6$ indicates a fair quality of this SPS-compacted polycrystalline sample. The onset of the rounded superconducting transition occurs at $T_{\mathrm{c}}=2.9(1) \mathrm{K}$, slightly lower than the value of $3.04 \mathrm{~K}$ deduced for single crystalline material.

The specific heat was measured on single crystals applying different magnetic fields $B \| c$ and $B \perp c$ (inset Fig. 8) as well as on the polycrystalline SPS sample. Further analysis of the $c_{p}(T, B)$ data is performed on the base of bulk specimen, since they reproduce the $B \| c$ results of single crystal (Fig. 8) and reveal a better statistic due to higher sample mass.

The overcritical specific heat is fitted in the temperature range $0.35-1.7 \mathrm{~K}$ to the ansatz $c_{p}=\gamma_{\text {tot }} T+\beta T^{3}$ (not 


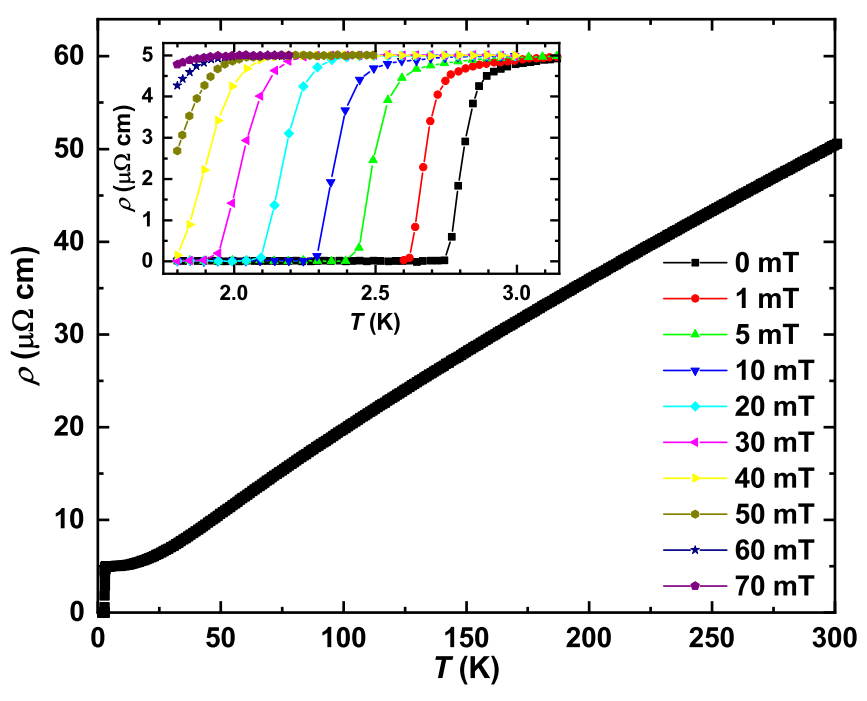

FIG. 7. Electrical resistivity $\rho(T)$ for SPS-compacted polycrystalline $\mathrm{TaSnS}_{2}$. Inset: $\rho(T)$ in different magnetic fields.

shown in Fig. 8) with $\gamma_{\text {tot }}=5.4(1) \mathrm{mJ} \mathrm{mol}^{-1} \mathrm{~K}^{-2}$ and $\beta=$ $0.64(2) \mathrm{mJ} \mathrm{mol}^{-1} \mathrm{~K}^{-4}$ [corresponds to an initial Debye temperature $\left.\theta_{\mathrm{D}}=229(2) \mathrm{K}\right]$.

The transition temperature midpoint $T_{\mathrm{c}}=2.88(2) \mathrm{K}$ (i.e., the middle of the falling flank of the anomaly) observed in $c_{p}$ measurements is in agreement with the $T_{\mathrm{c}}$ derived from the other properties. The steplike anomaly corresponding to the superconducting transition is rounded (Fig. 8), which is due to chemical inhomogeneities appearing in the specimen after SPS. The estimated specific heat jump for the sintered sample and single crystal is $\Delta c_{p} / \gamma T_{\mathrm{c}} \approx 1.2$ (obtained by a graphical equal-areas construction in a $c_{p} / T$ vs $T$ plot, i.e., entropy conserving) and is in fair agreement with the BCS model (1.43).

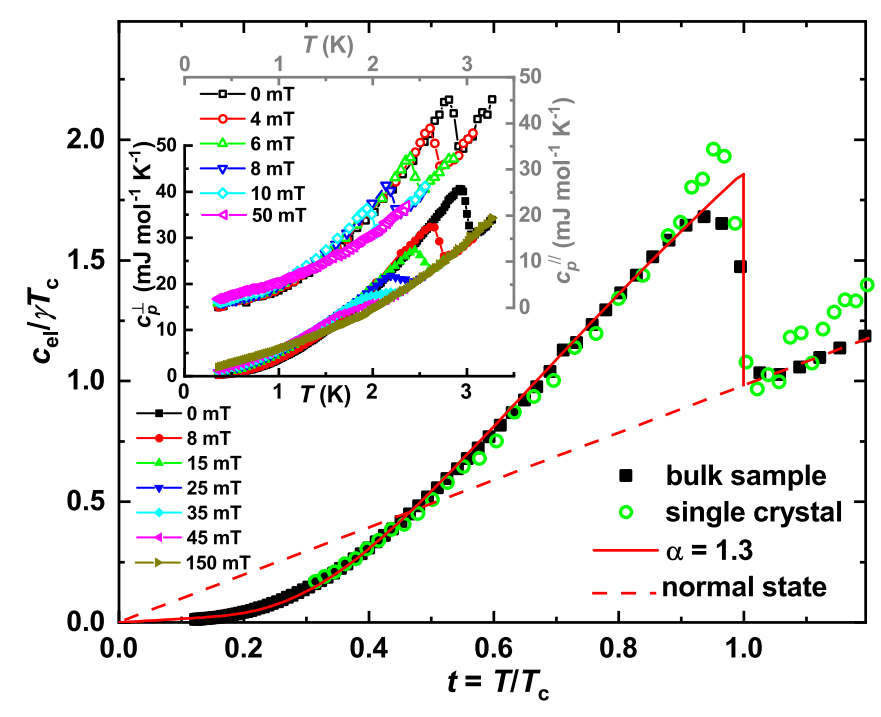

FIG. 8. Electronic specific heat $c_{\mathrm{el}} / \gamma T_{\mathrm{c}}$ versus $t=T / T_{\mathrm{c}}$ for $\mathrm{TaSnS}_{2}$ [midpoint $T_{\mathrm{c}}=2.88(2) \mathrm{K}$ ] and comparison with the $\alpha$ model [79]. Inset: Specific heat in various magnetic fields for $\mathrm{TaSnS}_{2}$ single crystals with the magnetic field applied $\| c$ (right scale) and $\perp c$ (left scale).
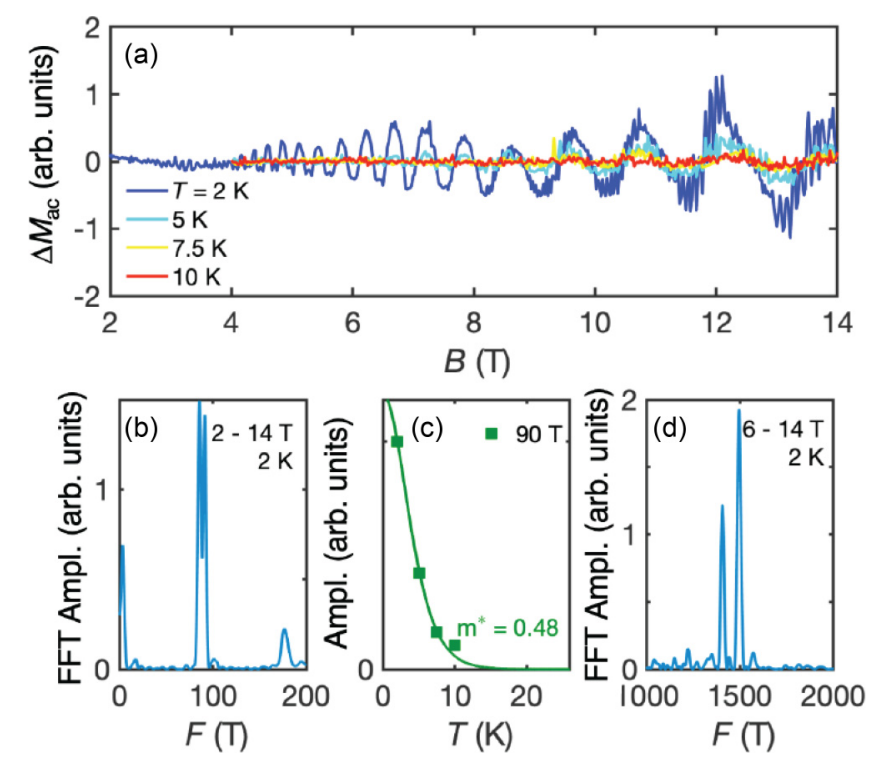

FIG. 9. Quantum oscillations in polycrystalline $\mathrm{TaSnS}_{2}$ (a) oscillatory component of the ac susceptibility (b) Fourier transform over 2-14 $\mathrm{T}$ window (c) decay of the oscillatory amplitude with increasing temperature at the field value $B_{\mathrm{osc}}=13.1 \mathrm{~T}$ (dominated by the $90 \mathrm{~T}$ oscillation at this field value) and (d) Fourier transform over the field window 6-14 T.

After subtracting the phononic part $\left(\beta T^{3}\right)$, we obtain the electronic specific heat for polycrystalline sample and single crystal (Fig. 8). Both curves excellently match for $T<0.8 T_{\mathrm{c}}$ and are well described by the $\alpha$ model for $\alpha \equiv \Delta(0) / k_{\mathrm{B}} T_{\mathrm{c}} \approx$ 1.3. Although the value of $\alpha$ is below that for the weakcoupling limit of the BCS theory $\left(\alpha_{\mathrm{BCS}}=1.76\right.$ [79]), there are no visible indications for possible multigap superconductivity in our data. We thus conclude that $\mathrm{TaSnS}_{2}$ is a weak-coupling $s$-wave superconductor.

\section{Quantum oscillations and electronic structure}

The small effective masses of semimetals tend to result in a pronounced de Haas-van Alphen (dHvA) effect [80]. Here, the frequency $F$ of the oscillation is related with the extremal Fermi surface (FS) cross section $S_{\text {ext }}$ perpendicular to the applied magnetic field by the Onsager relation $F=\hbar S_{\text {ext }} / 2 \pi e$. The temperature damping of the oscillation amplitude $A(T)$ at a fixed magnetic field $B_{\text {osc }}$ is given by the Lifshitz-Kosevitch formular $A(T)=\chi / \sinh (\chi)$ with $\chi=$ $2 \pi^{2} m^{*} k_{\mathrm{B}} T / e \hbar B_{\mathrm{osc}}$ [80]. This behavior allows us to extract the effective cyclotron mass $m^{*}$ of the charge carriers. Figure 9(a) shows well-resolved quantum oscillations (QO) in the ac magnetization as a function of applied dc field of the polycrystalline sample. Since the $\mathrm{dHvA}$ signal is periodic in $1 / B$, the field axis is inverted and a Fourier transform (FFT) performed over a window of 2-14T [Fig. 9(b)] and 6-14 T [Fig. 9(d)]. The FFT reveals two sets of QO frequencies, each one split into two: One at 90/100 $\mathrm{T}$ and one at 1400 and $1500 \mathrm{~T}$. The beating patterns observable in Fig. 9(a) are a result of the splitting of the lower frequencies. The fact that we found pronounced QOs even in polycrystalline powder certifies the high sample quality. 
Given the quick decay of the amplitude with temperature, we can get a rough estimate of the effective mass of the orbit corresponding to the dominant $90 \mathrm{~T}$ frequency. The temperature dependence of the amplitude at $13.1 \mathrm{~T}$ is shown in Fig. 9(c). With this, we estimate the effective mass to be $\approx 0.48 m_{e}$.

We can also compare the $\mathrm{QO}$ frequencies we found with the ones observed in the related material $\mathrm{TaPbS}_{2}$ and pristine $2 \mathrm{H}-\mathrm{TaS}_{2}$, in order to get an idea which pockets or orbits are responsible for the observed QOs. In $\mathrm{TaPbS}_{2}$ a rather large FS was found by density-functional theory (DFT) and confirmed by angle-resolved photoemission spectroscopy (ARPES), consisting of a hexagonal center connected to another, almost cylindrical surface along the $\mathrm{H}-\mathrm{K}$ line at the corner of the Brillouin zone (BZ) [81,51]. Within this there are also a number of cylindrical nested FS pockets. Around the $\Gamma$ point, two nested pillow-shaped FS pockets are found. Experimentally, QO frequencies between 6 and $1200 \mathrm{~T}$ with an angular dependence in agreement with the predicted almost 2D nature (i.e., cylindrical shape of the FS) were observed.

In the pristine compound $2 \mathrm{H}-\mathrm{TaS}_{2}$, two large 2D FS both located at the center and the corner of the $\mathrm{BZ}$ are found by electronic structure calculations [82]. These common quasi2D structures of the two closely related materials lead us to search for similar FS pockets in $\mathrm{TaSnS}_{2}$. As the calculations below show, the small, almost cylindrical FS sheets at the corner of the $\mathrm{BZ}$ in $\mathrm{TaSnS}_{2}$ (similar to those observed in $\mathrm{TaPbS}_{2}$ ) could appear if the Fermi energy is slightly shifted to the electron-doped region. This could explain the lower pair of $\mathrm{QO}$ frequencies.

Since the QOs are observed in the polycrystalline sample it is difficult to assign FS pockets with observed frequencies, because of the expected angular dependence of the frequencies and arbitrary field directions in the polycrystal. A full angulardependent study on single crystals would give further insight if the observed FS pockets have quasi 2D or 3D character. We observed dHvA oscillations in fields of several Tesla on the largest presently available single crystal $(m \approx 30 \mathrm{mg})$. They were more or less consistent with the slow ones observed on the much heavier polycrystalline sample. However, the obtained data were noisy, indicating the sample mass to be too low. Therefore, to perform such a study syntheses of large enough crystals should be further optimized.

The band structure of $\mathrm{TaSnS}_{2}$ has been reported before in the context of ARPES results [38] and very recently upon the discussion of $\mathrm{TaSnS}_{2}$ as a nodal line semimetal [47]. In order to estimate the extreme orbits observed in the dHvA experiments we performed highly accurate and well converged LAPW calculations. Figure 10 shows the FS of $\mathrm{TaSnS}_{2}$ which consist of an ellipsoid sheet (holelike) and-due to symmetry-doubly degenerated open hexagonal sheet (electronlike), which nearly shows no $k_{\mathrm{z}}$ dispersion.

The extremal cross section of the hole sheet in the $\Gamma-\mathrm{K}$ A plane (field perpendicular to that plane) is about $1700 \mathrm{~T}$. Shifting the Fermi level up by $4 \mathrm{mRy}(54 \mathrm{meV})$ would bring this value to $1500 \mathrm{~T}$. In contrast, shifting the $E_{\mathrm{F}}$ down would increase the extremal area and at some point even create an open orbit where no QO would be observable. Similar discrepancies between calculation and experiment have been observed before [83]. One possible explanation could be the

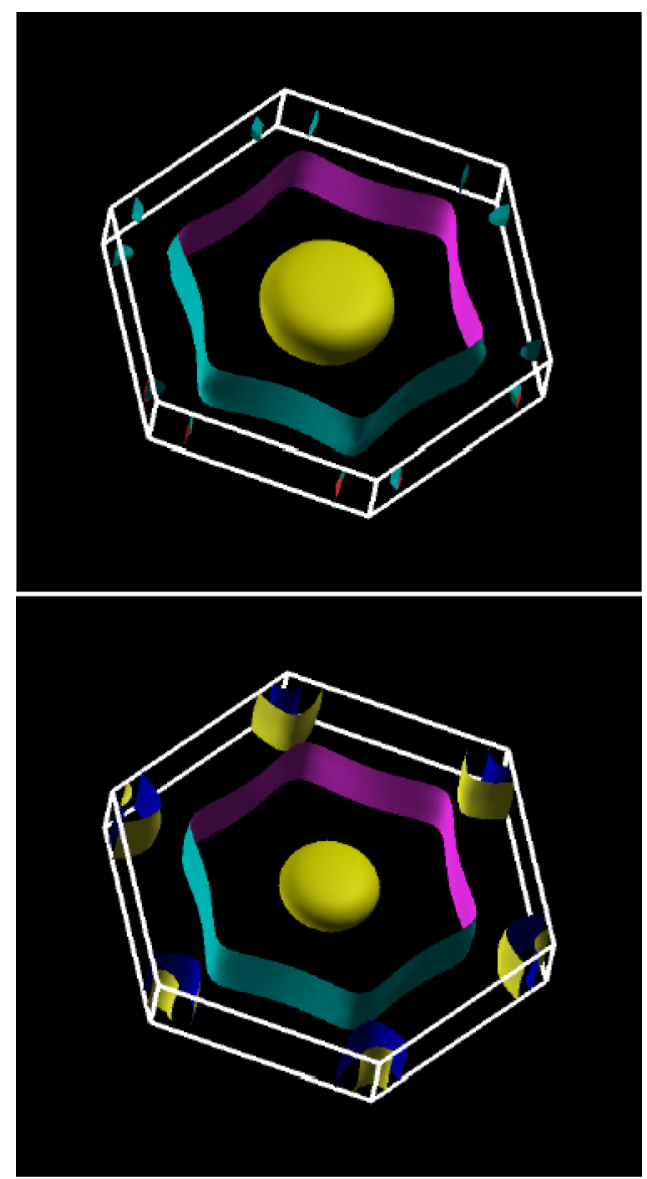

FIG. 10. Fermi surface of $\mathrm{TaSnS}_{2}$ (a) and the same with shift of the Fermi level by $20 \mathrm{mRy}(=0.27 \mathrm{eV})(\mathrm{b})$. The hole sheet in the $\Gamma-\mathrm{K}-\mathrm{A}$ plane is given in yellow color.

well known limitation of DFT caused by the error introduced by the self interaction. This will lead to the overestimation of the delocalization of atomic orbitals and thus should overestimate the interplane interactions.

However, the small orbits of about $100 \mathrm{~T}$ would not be consistent with this FS without shifting the Fermi level $E_{\mathrm{F}}$. As one can see in Fig. 10(b), small pockets close to the $\mathrm{K}$ point of the Brillouin zone will open and the size of these orbits depends on the shift of $E_{\mathrm{F}}$. As discussed in Ref. [47] for $\mathrm{TaSnS}_{2}$ this could lead to the line nodes under some conditions. For $\mathrm{TaPbS}_{2}$ the band structure and the observed dHvA oscillations are discussed in great detail [81]. The studies confirm that the position of $E_{\mathrm{F}}$ is crucial in the formation of line nodes around $\mathrm{K}$ and $\Gamma$. A relatively large shift of about $20 \mathrm{mRy}(0.27 \mathrm{eV})$ would be required for $\mathrm{TaSnS}_{2}$ to get a match with some of the dHvA oscillations observed. While the disagreement could be at least partially due to failures of DFT, this appears to be somewhat large and inconsistent. Eventually it may also be a hint towards a small deviation from stoichiometry, because electron doping could have a similar effect to $E_{\mathrm{F}}$. Another possibility could be a small deviation of the $c / a$ ratio of the polycrystalline samples compared to the single crystal due to slightly different compositions, which could produce relative shifts of the bands to each other. 


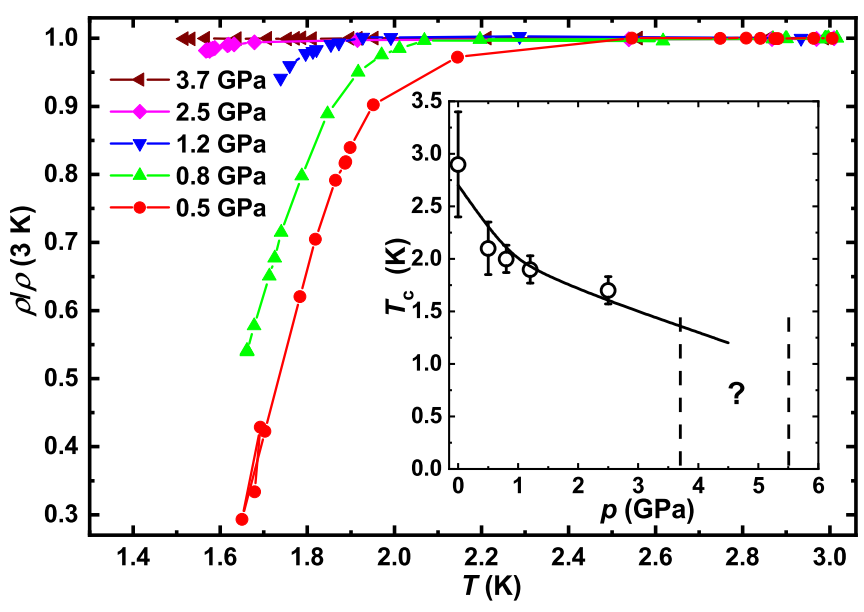

FIG. 11. The in situ resistivity measurements at high pressures reveal the pressure dependence of $T_{\mathrm{c}}$ (inset). No superconducting transition was observed down to $1.5 \mathrm{~K}$ for pressures of 3.7 and $5.5 \mathrm{GPa}$ (not shown), so that we assume $T_{\mathrm{c}}$ to be in the temperature range $0-1.5 \mathrm{~K}$. The solid line is a guide to the eye.

\section{E. Pressure effect on superconductivity and structure of $\mathrm{TaSnS}_{2}$}

Electrical resistivity measurements under application of high pressure reveal a continuous decrease of the superconducting $T_{\mathrm{c}}$ with pressure increase (inset to Fig. 11). This behavior is contrary to the related misfit layered $(\mathrm{SnS})_{1.15}\left(\mathrm{TaS}_{2}\right)$ compound [70]. The decrease of $T_{\mathrm{c}}$ in $\mathrm{TaSnS}_{2}$ results primarily from a weakening of electron-phonon coupling due to the stiffening of the crystalline lattice (shift of phonon spectrum to higher frequencies) upon compression [84]. The onset of SC can be observed as small resistivity drop at the temperature below $1.8 \mathrm{~K}$ as pressure increases up to the $2.5 \mathrm{GPa}$ while no indication for $\mathrm{SC}$ is observed by cooling down to $1.5 \mathrm{~K}$ at further pressure increase above $3 \mathrm{GPa}$ (Fig. 11).

The suppression of superconducting state at pressures above $3 \mathrm{GPa}$ might be associated with onset of structural phase transition indicated by emergent asymmetry (lowfrequency shoulder) of the Raman peak located at $\sim 400 \mathrm{~cm}^{-1}$ as pressure increases above $3 \mathrm{GPa}$ and its split with further pressure increase (Fig. 12). To clarify the structure of the $\mathrm{TaSnS}_{2}$ high-pressure phase, high resolution synchrotron $\mathrm{x}$ ray diffraction studies are required.

\section{CONCLUSIONS}

The fully-intercalated TMD compound $\mathrm{TaSnS}_{2}$ was synthesized by powder metallurgical methods and further compacted by SPS. Single crystals of a size up to $1 \times 1 \times 0.1 \mathrm{~cm}$ were grown by chemical vapor transport. Single crystal $x$-ray diffraction confirmed the earlier reported [34], [35] space group $P 6_{3} / m m c$ with the lattice parameters $a=3.30648$ (1) $\AA$ and $c=17.4416(1) \AA$. No indications for a possible structural phase transition were observed below $300 \mathrm{~K}$.

Measurements of the magnetization, specific heat, and resistivity show superconductivity at $T_{\mathrm{c}}=3.0(1) \mathrm{K}$. The upper critical fields in both directions (i.e., $B \perp c$ and $B \| c$ ) evidence a moderate anisotropy in $\mathrm{TaSnS}_{2}$. The observed specific heat

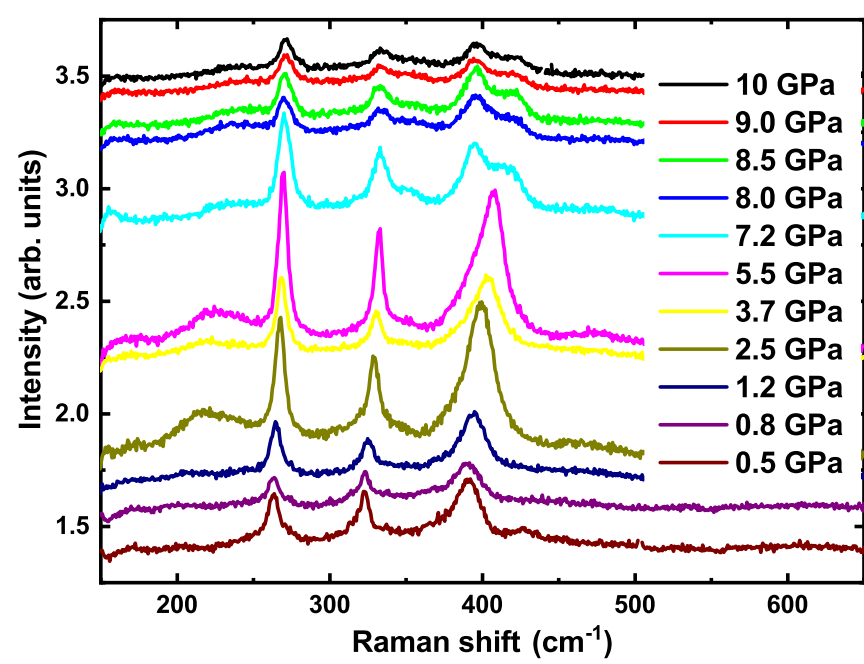

FIG. 12. Raman spectra in the pressure range 0.5-10 GPa showing a peak splitting at $400 \mathrm{~cm}^{-1}$.

jump $\Delta c_{\mathrm{p}} / \gamma T_{\mathrm{c}} \approx 1.2$ and energy gap ratio $\Delta(0) T / k_{\mathrm{B}} T_{\mathrm{c}} \approx 1.3$ agree fairly well with the values (i.e., 1.43 and 1.76 , respectively) predicted by weak coupling BCS theory. Together with the Ginzburg-Landau parameter $\kappa_{\mathrm{GL}, a b} \approx 3>1 / \sqrt{2}$, we conclude $\mathrm{TaSnS}_{2}$ to be a weakly coupled type-II BCS $s$-wave superconductor.

De Haas-van Alphen quantum oscillations, revealing an effective mass of about $0.5 m_{e}$, have been observed in $\mathrm{TaSnS}_{2}$ polycrystalline sample. These oscillations originate probably from a small Fermi-surface pocket at the corner of the Brillouin zone and an ellipsoid in the center as comparisons with the band-structure calculations show.

In contrast to other nodal line semimetals the application of pressure leads to a strong suppression of the superconductivity in $\mathrm{TaSnS}_{2}$. A pressure of $p_{\mathrm{c}}=3.7 \mathrm{GPa}$ is sufficient to shift the superconducting transition temperature below $T=1.5 \mathrm{~K}$. Such a comparatively strong pressure dependence could originate from a pressure-induced structural phase transition, which is seemingly visible in the pressure dependent Raman study.

Our results demonstrate that $\mathrm{TaSnS}_{2}$ is an interesting superconductor with a moderate anisotropy. So far the impact of the topological line nodes present in the band structure on the physical properties and especially on the superconductivity has not been fully clarified which certainly triggers further studies on $\mathrm{TaSnS}_{2}$.

\section{ACKNOWLEDGMENTS}

This work is performed within DFG (Deutsche Forschungsgemeinschaft) Grant No. 325295543. We thank M. Schmidt for his support during chemical vapor transport syntheses and Yu. Grin for his steady interest in this work. Technical support of H. Rave and C. Klausnitzer is acknowledged. The authors are grateful to R. Koban for physical measurements. 
[1] N. B. Hannay, T. H. Geballe, B. T. Matthias, K. Andres, P. Schmidt, and D. MacNair, Phys. Rev. Lett. 14, 225 (1965).

[2] E. A. Antonova, S. A. Medvedev, and I. Y. Shebalin, J. Exp. Theor. Phys. (Rus) 30, 181 (1970).

[3] F. R. Gamble, F. J. DiSalvo, R. A. Klemm, and T. H. Geballe, Science 168, 568 (1970).

[4] R. A. Klemm, Layered Superconductors (Oxford Science Publications, Oxford, 2012).

[5] J. G. Bednorz and K. A. Müller, Z. Phys. B 64, 189 (1986).

[6] Y. Hidaka, Y. Enomoto, M. Suzuki, M. Oda, A. Katsui, and T. Murakami, Jpn. J. Appl. Phys. 26, 1133 (1987).

[7] Y. Iye, T. Tamegai, H. Takeya, and H. Takei, Jpn. J. Appl. Phys. 26, L1057 (1987).

[8] T. K. Worthington, W. J. Gallagher, and T. R. Dinger, Phys. Rev. Lett. 59, 1160 (1987).

[9] T. R. Dinger, T. K. Worthington, W. J. Gallagher, and R. L. Sandstrom, Phys. Rev. Lett. 58, 2687 (1987).

[10] Z.-S. Wang, H.-Q. Luo, C. Ren, and H.-H. Wen, Phys. Rev. B 78, 140501 (2008).

[11] M. Fang, J. Yang, F. F. Balakirev, Y. Kohama, J. Singleton, B. Qian, Z. Q. Mao, H. Wang, and H. Q. Yuan, Phys. Rev. B 81, 020509(R) (2010).

[12] P. C. Canfield and S. L. Bud'ko, Annu. Rev. Condens. Matter Phys. 1, 27 (2010).

[13] M. Nagao, A. Miura, S. Demura, K. Deguchi, S. Watauchi, T. Takei, Y. Takano, N. Kumada, and I. Tanaka, Solid State Commun. 178, 33 (2014).

[14] T. Yoshida, S. Ideta, T. Shimojima, W. Malaeb, K. Shinada, H. Suzuki, I. Nishi, A. Fujimori, K. Ishizaka, S. Shin et al., Sci. Rep. 4, 7292 (2014).

[15] Y. Mizuguchi, A. Miyake, K. Akiba, M. Tokunaga, J. Kajitani, and O. Miura, Phys. Rev. B 89, 174515 (2014).

[16] Y. C. Chan, K. Y. Yip, Y. W. Cheung, Y. T. Chan, Q. Niu, J. Kajitani, R. Higashinaka, T. D. Matsuda, Y. Yanase, Y. Aoki et al., Phys. Rev. B 97, 104509 (2018).

[17] R. A. Klemm, Physica C 514, 86 (2015).

[18] K. Onabe, M. Naito, and S. Tanaka, J. Phys. Soc. Jpn. 45, 50 (1978).

[19] J. Wilson, F. D. Salvo, and S. Mahajan, Adv. Phys. 24, 117 (1975).

[20] J. M. E. Harper, T. H. Geballe, and F. J. DiSalvo, Phys. Rev. B 15, 2943 (1977).

[21] E. Revolinsky, G. Spiering, and D. Beerntsen, J. Phys. Chem. Solids 26, 1029 (1965).

[22] M. van Maaren and H. Harland, Phys. Lett. A 29, 571 (1969).

[23] K. Yokota, G. Kurata, T. Matsui, and H. Fukuyama, Phys. B: Condens. Matter 284-288, 551 (2000).

[24] X. Li, M. Zhou, and C. Dong, Supercond. Sci. Technol. 32, 035001 (2018).

[25] M. Ikebe, N. Kobayashi, K. Katagiri, and Y. Muto, Physica B+C 105, 435 (1981).

[26] B. Dalrymple and D. Prober, J. Low Temp. Phys. 56, 545 (1984).

[27] M. S. Whittingham, Prog. Solid State Chem. 12, 41 (1978).

[28] F. R. Gamble, J. H. Osiecki, and F. J. DiSalvo, J. Chem. Phys. 55, 3525 (1971).

[29] M. H. Zhou, X. C. Li, and C. Dong, Supercond. Sci. Technol. 31, 065001 (2018).
[30] K. E. Wagner, E. Morosan, Y. S. Hor, J. Tao, Y. Zhu, T. Sanders, T. M. McQueen, H. W. Zandbergen, A. J. Williams, D. V. West et al., Phys. Rev. B 78, 104520 (2008).

[31] L. Li, X. Zhu, Y. Sun, H. Lei, B. Wang, S. Zhang, X. Zhu, Z. Yang, and W. Song, Physica C: Supercond. 470, 313 (2010).

[32] R. Eppinga and G. Wiegers, Physica B+C 99, 121 (1980).

[33] E. A. Marseglia, Int. Rev. Phys. Chem. 3, 177 (1983).

[34] R. Eppinga and G. Wiegers, Mater. Res. Bull. 12, 1057 (1977).

[35] A. van der Lee and G. Wiegers, Mater. Res. Bull. 25, 1011 (1990).

[36] F. J. Di Salvo, G. W. Hull, L. H. Schwartz, J. M. Voorhoeve, and J. V. Waszczak, J. Chem. Phys. 59, 1922 (1973).

[37] R. Eppinga, G. Wiegers, and C. Haas, Physica B+C 105, 174 (1981).

[38] J. Dijkstra, E. A. Broekhuizen, C. F. van Bruggen, C. Haas, R. A. de Groot, and H. P. van der Meulen, Phys. Rev. B 40, 12111 (1989).

[39] R. Eppinga, G. A. Sawatzky, C. Haas, and C. F. van Bruggen, J. Phys. C: Solid State Phys. 9, 3371 (1976).

[40] R. H. Herber and R. F. Davis, J. Chem. Phys. 63, 3668 (1975).

[41] R. H. Herber and R. F. Davis, J. Chem. Phys. 65, 3773 (1976).

[42] P. Gentile, D. Driscoll, and A. Hockman, Inorg. Chim. Acta 35, 249 (1979).

[43] R. H. Herber, F. J. DiSalvo, and R. B. Frankel, Inorg. Chem. 19, 3135 (1980).

[44] A. C. Gossard, F. J. d. Salvo, and H. Yasuoka, Phys. Rev. B 9 , 3965 (1974).

[45] G. Y. Guo and W. Y. Liang, J. Phys. C: Solid State Phys. 20, 4315 (1987).

[46] P. Blaha, J. Phys.: Condens. Matter 3, 9381 (1991).

[47] D.-Y. Chen, Y. Wu, L. Jin, Y. Li, X. Wang, J. X. Duan, J. Han, X. Li, Y.-Z. Long, X. Zhang, D. Chen, and B. Teng, Phys. Rev. B 100, 064516 (2019).

[48] Y. Qi, P. G. Naumov, M. N. Ali, C. R. Rajamathi, W. Schnelle, O. Barkalov, M. Hanfland, S.-C. Wu, C. Shekhar, Y. Sun et al., Nat. Commun. 7, 11038 (2016).

[49] Y. Fang, Q. Dong, J. Pan, H. Liu, P. Liu, Y. Sun, Q. Li, W. Zhao, B. Liu, and F. Huang, J. Mater. Chem. C 7, 8551 (2019).

[50] J. J. Gao, J. G. Si, X. Luo, J. Yan, Z. Z. Jiang, W. Wang, C. Q. Xu, X. F. Xu, P. Tong, W. H. Song et al., J. Phys. Chem. C 124, 6349 (2020).

[51] G. Bian, T.-R. Chang, R. Sankar, S.-Y. Xu, H. Zheng, T. Neupert, C.-K. Chiu, S.-M. Huang, G. Chang, I. Belopolski et al., Nat. Commun. 7, 10556 (2016).

[52] S.-Y. Guan, P.-J. Chen, M.-W. Chu, R. Sankar, F. Chou, H.-T. Jeng, C.-S. Chang, and T.-M. Chuang, Sci. Adv. 2, e1600894 (2016).

[53] B.-T. Wang, P.-F. Liu, J.-J. Zheng, W. Yin, and F. Wang, Phys. Rev. B 98, 014514 (2018).

[54] P.-J. Chen, T.-R. Chang, and H.-T. Jeng, Phys. Rev. B 94, 165148 (2016).

[55] STOE Powder Software, WinXPow (version 2), Darmstadt, STOE and Cie GmbH, 2001.

[56] Bruker AXS Inc., DOC-M86-EXX190 D8 QUEST User Manual (2012).

[57] Bruker AXS Inc., DOC-M86-EXX229 APEX3 Software User Manual (2016).

[58] L. Akselrud and Y. Grin, J. Appl. Crystallogr. 47, 803 (2014). 
[59] G. Carter, L. Bennett, and D. Kahan, in Progress in Materials Science, edited by B. Chalmers, J. Christian, and T. Massalski (Pergamon Press, Oxford, 1977), Vol. 81, pp. 779-780.

[60] P. Blaha, K. Schwarz, F. Tran, R. Laskowski, G. K. H. Madsen, and L. D. Marks, J. Chem. Phys. 152, 074101 (2020).

[61] J. P. Perdew and Y. Wang, Phys. Rev. B 45, 13244 (1992).

[62] J. P. Perdew, A. Ruzsinszky, G. I. Csonka, O. A. Vydrov, G. E. Scuseria, L. A. Constantin, X. Zhou, and K. Burke, Phys. Rev. Lett. 100, 136406 (2008).

[63] http://www.goldensoftware.com/products/surfer/surfer.shtml.

[64] S. A. Khan, J. M. Reynolds, and R. G. Goodrich, Phys. Rev. 163, 579 (1967).

[65] See Supplemental Material at http://link.aps.org/supplemental/ 10.1103/PhysRevB.102.214501 for results of powder XRD phase analysis and magnetic ac susceptibility measurement.

[66] R. Gross and A. Marx, Festkörperphysik (Oldenbourg Verlag, München, 2012).

[67] D. E. Prober, R. E. Schwall, and M. R. Beasley, Phys. Rev. B 21, 2717 (1980).

[68] M. N. Ali, Q. D. Gibson, T. Klimczuk, and R. J. Cava, Phys. Rev. B 89, 020505(R) (2014).

[69] M. Ikebe, K. Katagiri, K. Noto, and Y. Muto, Physica B+C 99, 209 (1980).

[70] R. Sankar, G. Peramaiyan, I. Panneer Muthuselvam, C.-Y. Wen, X. Xu, and F. C. Chou, Chem. Mater. 30, 1373 (2018).

[71] L. Fang, Y. Wang, P. Y. Zou, L. Tang, Z. Xu, H. Chen, C. Dong, L. Shan, and H. H. Wen, Phys. Rev. B 72, 014534 (2005).
[72] D. Bhoi, S. Khim, W. Nam, B. S Lee, C. Kim, B.-G. Jeon, B. H. Min, S. Park, and K. Kim, Sci. Rep. 6, 24068 (2016).

[73] R. Coleman and S. Hillenius, Physica B+C 105, 428 (1981).

[74] R. A. Klemm, A. Luther, and M. R. Beasley, Phys. Rev. B 12, 877 (1975).

[75] Y. Iye and S. I. Tanuma, Phys. Rev. B 25, 4583 (1982).

[76] A. Chaiken, M. S. Dresselhaus, T. P. Orlando, G. Dresselhaus, P. M. Tedrow, D. A. Neumann, and W. A. Kamitakahara, Phys. Rev. B 41, 71 (1990).

[77] K. Holczer, O. Klein, G. Grüner, J. D. Thompson, F. Diederich, and R. L. Whetten, Phys. Rev. Lett. 67, 271 (1991).

[78] M. Baenitz, M. Kraus, S. Gärtner, H. M. Vieth, H. Werner, R. Schlögl, W. Krätschmer, M. Kanowski, and K. Lüders, in Electronic Properties of High-Tc Superconductors, edited by H. Kuzmany, M. Mehring, and J. Fink (Springer Berlin Heidelberg, Berlin, Heidelberg, 1993), pp. 475-481.

[79] D. C. Johnston, Supercond. Sci. Technol. 26, 115011 (2013).

[80] D. Shoenberg, Magnetic Oscillations in Metals (Cambridge University Press, Cambridge, UK, 1984).

[81] X. Xu, Z. Kang, T.-R. Chang, H. Lin, G. Bian, Z. Yuan, Z. Qu, J. Zhang, and S. Jia, Phys. Rev. B 99, 104516 (2019).

[82] G. Wexler and A. M. Woolley, J. Physics C: Solid State Phys. 9, 1185 (1976).

[83] I. I. Mazin and J. Kortus, Phys. Rev. B 65, 180510(R) (2002).

[84] J. Schilling, in Handbook of High-Temperature Superconductivity, edited by J. R. Schrieffer (Springer, Berlin, 2007), pp. 427-462. 